\title{
A Survey about Fusion of Blockchain Technology in Various Application Realms
}

\author{
G R Karpagam ${ }^{1}$ \\ \{grk.cse@psgtech.ac.in $\left.{ }^{1}\right\}$ \\ Professor, Department of Computer Science and Engineering, PSG College of Technology, Coimbatore, India ${ }^{1}$
}

\begin{abstract}
Blockchains have recently received an abundance attention as they provide a decentralized approach to value creation and management. Many financial institutions, banks, business sectors, public sectors, cyber-security, education and even healthcare sectors have started to incorporate Blockchains to improve safe, secure, and efficient services. The objective of the survey is to focus on providing incorporation of blockchain applications over different domains. These domain include business sector, government sectors, medical and healthcare, royalty, energy sector, IoT, cybersecurity, web community and education applications. The survey affords a concise overview for blockchain-interested individuals and organizations. We expect that our study will encourage further researchers to investigate and implement various applications using Blockchain Technology.
\end{abstract}

Keywords: Blockchains, Decentralized ledger, Immutable, Cryptocurrency, Healthcare, Business, Energy.

\section{Introduction}

Blockchains are now being debated in the news around the world. They have already been implemented as decentralized solutions to fraud-resistant computing without a trusted authority in many applications from different domains. A blockchain is a distributed decentralized ledger which appends log of time-stamped records that are cryptographically protected against tampering and revision of any transactions. Since concept of blockchain is introduced for Bitcoin transactions, for its features the technology can be entrenched among various domains. The objective of this survey exemplifies blockchain incorporations indifferent domains along with real-time examples. The areas includes business sector, government sectors [1], medical and healthcare [2], royalty [3], energy sector [4], IoT [5], cybersecurity [6], web community [7] and education applications [8]. With blockchain, healthcare records can be combined and be tracked patients electronic health records (EHR), and personal health records (PHR) for treatments [2] [9]. Some real-implementations like FollowMy vote Dapp to ensure secure and trusted voting system [10]. BlockcertsDapp to protect student's credential records which ensure easy tracking and tamper-proof of credentials [11]. Framatrust in healthcare used to track the drugs origin as raw materials to selling of drug in pharmaceuticals [12]. The Blockchain technology has attainment implementation over various domains. The survey accomplishes wide discussion of incorporating blockchain along with problems rectification and real-time examples, which are investigation gap untouched in prior survey papers.

\section{Blockchain Applications}

As communities are very active, multitudinous blockchains have emerged day-by-day for its features. A consortium block-chain is soundly applied for business applications that ensure centrally be secured, transparent, and permissioned environment. At present, a smart contract-based ethereum network framework is developed for supporting consortium block-chain [13]. However, Hyperledger is an emerging business consortium blockchain framework [14]. As a technology blockchain is deployed in several fields to enhance existing aspects. In this section, various applications are approximately categorized into business in section 2.1, government applications in section 2.2, medical and healthcare in section 2.2, education in section 4.4 , royalty in section 4.5 , IoT in section 4.6 , cybersecurity in section 4.7 , human resources in section 4.8 , Wils and Inheritance in section 4.9 and section 4.10 investigates about deployment of blockchain other than abovementioned criteria. Figure: 8 illustrate various application criteria of Blockchain Technology. 


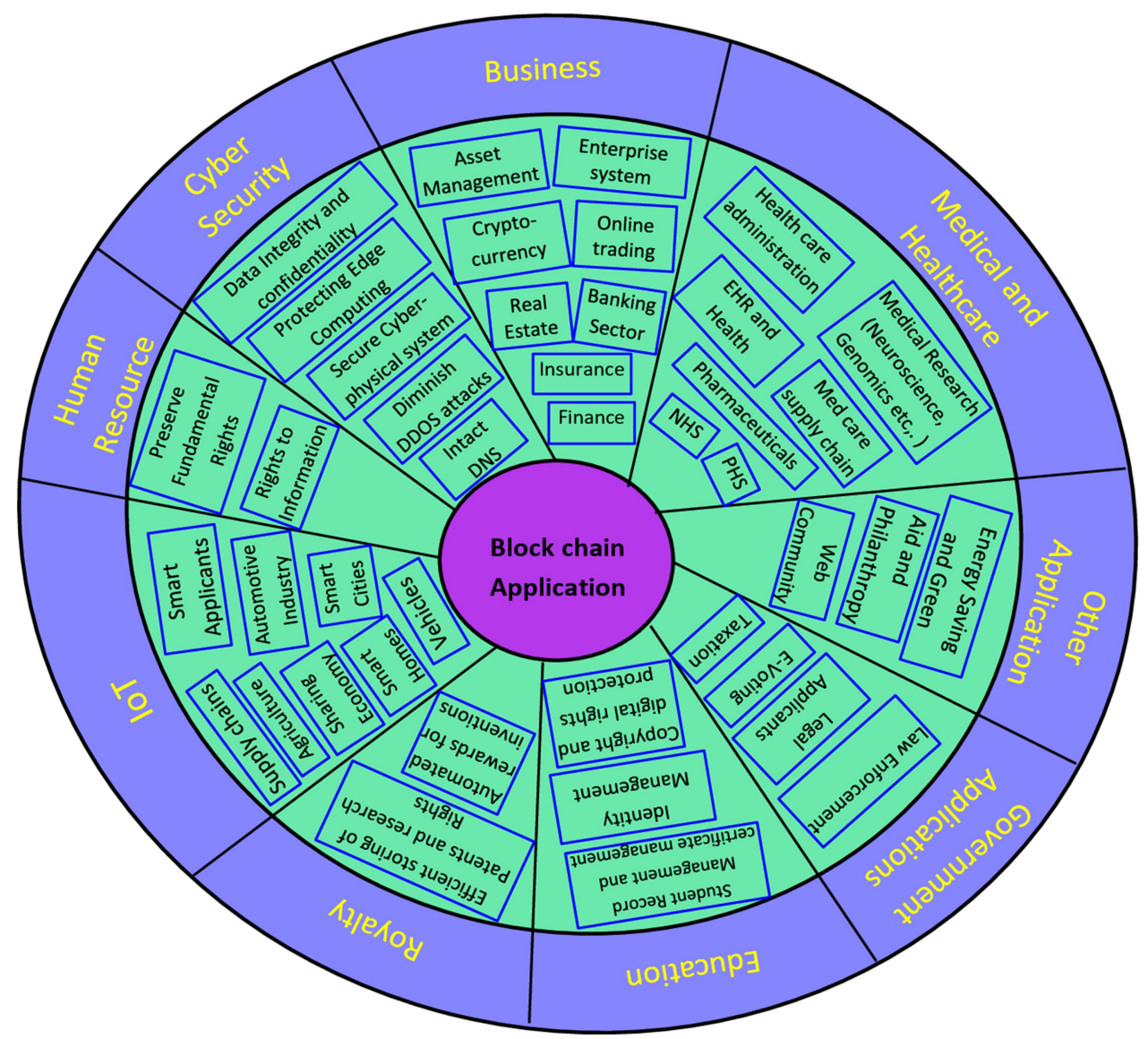

Figure 1: Blockchain Applications

\subsection{Blockchain in Business}

\subsubsection{Cryptocurrency}

At present, paper currency or coin-based currency is in regular use around worldwide. The currency or fiat currency values are assured and fixed by the government. Eg US Dollar, Great Britain Pound, Indian Rupees, Indonesia rupiah, European euro, and Swiss franc, etc. Commodity money across the world is backed by tradable resources, namely Gold and silver. But, Cryptocurrency is not fit for the aforementioned categories. Cryptocurrency is a digital currency medium to make transactions more secure and transparent. At the time December 2017, 1300 cryptocurrency existed already, which have a total market capitalization of USD $431,029,932,585$. Currently, Bitcoin cryptocurrency is most widely and successfully implemented for digital transactions which have a capitalization of approximately $\$ 24,747,300,000$. The fiat currency is used to measure the value of cryptocurrency. More types of cryptocurrencies are being generated for specific usage. Eg. Ether $(\mathrm{ETH})$ is used for ethereum network transactions. However, Ethereum and Ripple is a virtual currency, which has been used for enterprise transactions. An altcoin is an endorsed feature of Bitcoin. Other cryptocurrencies are i) Litecoin It makes an open-source global payment system that is not been controlled by any central parties. However, Ethereum and Ripple is a virtual currency, which has been used for enterprise cryptocurrencies are i) Litecoin makes an open-source global payment system that is not been controlled by any central authority. ii) ZCash Provides more security and privacy for financial transaction, iii) Dash makes untraceable transaction. iv) Ripple- Enables real-time cross-border payments at low cost and with end-to-end transparency. v) Monero (XMR) provides a private, secure and untraceable currency. vi) Bitcoin Cash (BCH) A type of Altcoin, vii) 
NEO (NEO) - A type of smart contracts transaction same of ETH. vii) Cardano it is used for Dapps and smart contracts platform, and viii) EOS (EOS) used for Bitshares digital currency exchange and social media platform [15].

\subsubsection{Financial Services}

Traditional financial systems highly depend on intermediates to mediate the financial process and resolve the existing conflicts. It leads to high intermediates cost, time consumption for processing intervenes work which naturally causes stress to complete our own work. To reduce these challenges, blockchain concepts along with Smart contract and Smart bonds are incorporated to automatically maintain abundant number of financial services. As it is a self-execute and self-maintain digital contract which automatically executes the paying process and made transaction based on pre-programming, we are not required middle-party for verification, and secure transaction. The Blockchain reduces barriers that are currently faced by finance industries are slow execution, frauds, vulnerability, and expensive cross-border transactions [16]. As, it having the potential to execute transparent, immutable and high speed payments, it gives a great benefits like incredible cost savings and high operational efficiencies. The first implementation of blockchain for payments system is introduced for restaurants and merchants by Taipei Fubon - A Commercial bank in Taiwan. Guardtime is a blockchain assisted company that has provided significant solutions for illegitimate practice in the financial industry such as reducing fraudulent and detecting data poisoning. Approximately 30 to 50 days has been taken for Know Your Customer (KYC) process to reach a satisfactory level. It creates a great deal for the existing financial system such as banks and government institutions. The present KYC system is suffering from duplication of labors and high cost. To resolve these issues, blockchain stores KYC information and edge off the need to ask customers again. Recently, Mastercard submits a patent contains a system of implementing private and semi-private blockchains for storing customer data like name, identification number, address, and tax details. Payroll and settlement clearance for public service transaction like buying a flight ticket, train tickets, buying property and selling, marriage registration, and patent management, etc., are requires series of complex actions for verifying and validating of the data provided by involved transaction parties. It formulas significant Turn Around Time (TAT). But, as we see the based blockchain settlements, it has been very faster and automatic execution that consequently reduces TAT eg. Automatic Pension System (APS). APS is a self-sustain autonomous contract grounded system that manages life-based pension schemes like collect funds from active customers, make settlements to beneficiaries according to contract. Setsoft is developed for an automatic pension scheme engaged over active customers and beneficiaries until death [17].

\subsubsection{Enterprise Transformation}

Blockchain is deployed to achieve smooth enterprise transformation example, postal operators (PO). PO for an enterprise system that acts as trusted authority over public. Postcoin is a type of colored coin of bitcoin. Postal Operators using Postcoin succeeding the dense retain network. Business opportunities for PO are supply chain management, identity management, and device management [18].

\subsubsection{Trading Platform}

Online trading is a great place for investors and clientele to trade and monitor shares, stocks, and commodities. However, drawbacks in online trading are entire system is controlled in hand of intermediaries with a double pending problem, risk of frauds, and lack of transparencies. These cons are reduced by deploying blockchain that offers a potential environment of interchanging of assets without the interference of intermediaries. It also enhances online assets authenticity and tractability by storing data in a permanent ledger. Everledger is an international startup organization that habits bitcoin as a mark to maintaining the authenticity of diamonds and related records of the diamond trade in a permanent digital ledger. ChainTrade is a blockchainbased physical platform for commodity trading to promote the simplified and fairness of trade processes [19].

\subsubsection{Banking Sector}

The first impact of blockchain technology was experienced over the banking sector. Bitcoin is the first cryptocurrency that uses blockchain technology for the transaction which has seen as a major threat for the banking business sector around the globe. Some experts are considering blockchain technology will take over numerous long-standing professional fields and businesses [20]. Traditional banking process consumes more time for multiple backend steps like verification, authentication for account creation, loan approval, and contract negotiations with multifarious parties. Blockchain based smart contracts reduce the speed for verification and authentication process. It also provides transparency of transactions that reduces double-spending, fraudulent 
and easily can detect the origin of the problem. Multiple banks already experimenting with a real-time Block-asa-Service offering by technical companies like IBM, Microsoft, R3 [21].Peters, G.W. and Panayi et al [20], analyzed and discuss about the automation of several niche aspects towards the banking sector like cash management, clearing settlements, client account reconciliation, Over the Contracts (OTC), cash settlements by governments, data loss reporting, etc.

\subsubsection{Asset Management}

Traditional asset management for the trading process experienced risky and expensive, particularly for cross-border transactions. Each peer involved in the process like customer, protector, broker settlement manager. It may expose significant inefficiencies for storing their data. In the case of implementing blockchain ledger for an asset management system which reduces occurrences of fraudulence, speed up the transactions, increase financial privacy and develop internationalized markets. It plays a prominent role in property ownership record tracking, property title management, a data-retrieval system of employees. For example, India has keenly looked over blockchain technology for providing solutions for problem arises over land registry. At present, property fraud is one of the biggest scams in India. In 2013, 181 property fraud cases are registered in New Delhi alone whereas it was followed by Mumbai that reserved a second place with 173 cases. To resolve these issues Governments of Telangana and Andhra Pradesh joined together with Swedish ChromewayStartup Company to implement blockchain for storing all-inclusive land registry records. Now, the land registry system is going extremely straightforward. ChromewayStartup system has a web app front-end and blockchain bankend based system [22]. The front-end serves perform user interactions while Chromeway perform monitoring and storing of data uses their own Postchainblockchain. According to the International Business Times: Postchain is developed to work systematically with wide-ranging platforms, which enable a seamless interaction with government systems.

One of the best innovations is the introduction of Postchain to maintain land registry by cryptographically secured digital fingerprints. Here, a hash is automatically generated from polygonial and geo-coordinates description of the land. The generated hash is intertwined with the land owners ID and resultant value generates a new hash, which will add into the blockchain. As, the resultant hash value is always unique, every owner would have a Unique ID and no one could able to tamper the records for its immutable property. J AChowdary, a IT Advisor and Special Chief Secretary of Andhra Pradesh from a cabinet of Chief Minister N. Chandrababu Naidu convey about this novel innovation. Blockchain technology governs the future era. It is having a lot of potentials to transform the entire economy. Government of Andhra Pradesh tied up with global start-ups like ChromeWay, to determine Proof- of-Concept (PoC - demonstrate the feasibility of blockchain in a real-world situation) for implementing blockchain within its own departments.

\subsubsection{Insurance}

Insurance is a process of protecting policyholders against financial loss. It is a contract, which exists between the insurance organization (the insurer) and policyholders. Insurance is a liability strategy used for protecting finance of policy holders for their own properties eg. Car insurance, Laptop Insurance, Health insurance, Life insurance, and travel insurance, etc [23]. If a particular person is insured on certain things, then the insurance company will be responsible to compensate for financial loss as per policy. The insurance premium is to be pay by policyholders as per policy. eg, a monthly basis, 3 months, 6 months, etc. If the policy term is finished, then policyholders will to renew or go through other insurance policies.

The problems faced by traditional system and problems rectified by blockchain system in business sector is shown in Table 1, and Real-Time implementation of blockchain in business sector is represented in Table 2. 
Table 1: Problem faced by traditional system and rectified by Blockchain system in Business sector

\section{Problems Faced by Traditional System}

Fraud - Centralized electronic digital fund transferring system is not secured. So, Cybercriminals steal asset information and modify the rental history through lands belonging to tenants.

Inaccurate market data - No reliable hub for consistently updating sellers and buyers list for real estate, shareholders share, market shares, etc. People inquire about the property via a centralized medium which may already undergo security compromised risk

Time intensive processes - Currently, more amount of moving papers pieces should be processed between inspection, loan approval, property approval, contingency release, unexpected error, and for a cash transaction.

No means to protect client dataDue to a lack of cybersecurity, clients' personal information is unveiled by various parties, leads to system vulnerability.

Financing difficulties - Centralized financial services are extremely bureaucratic that slows down the entire transaction. Eg. Securing a Home loan with low credit scores

Inefficiency - Insurance policy having lots of paper works like accurate inspection, moving of papers file from clerk to manager, manager to account department and vice versa that consumes lots of time to complete an entire transaction.

Lack of Security - Due to centralization, Cybercriminals can easily hack information and crash the server system.

Higher overhead - Lots of paper works wants to be processed for each transaction. It needs more humans intervention for each paperwork, that leads to more payments cost for mediates. Lots of errors, fraud are ascended in the centralization system.

Illegal transaction: No transparency is conserved during transaction. Chances are existed to mutate financial records, and prevail illegal transaction.

Traditional systems have a hole in security whicht paves a way to ensue fraudulent during clearing settlements, cash management, and client account reconciliation.

Intermediaries cause with double pending problem, frauds, due to lack of transparencies in online trading

\subsection{Blockchain for Government Applicants}

\subsubsection{E-Voting}

Main activities involved in the voting process are i) Election administrators: administrators are responsible for building election ballot using Dapps (decentralized apps). An administrator creates a smart contract with information like eligible qualification required for candidate register for voting, voting ballots, ballot district,
Real-time Immutable Data - If once ownership, rental details or any records related to assets transactions are stored, and then it can't be changed in the blockchain. So, no one can able to tamper the data.

Trustless - Real estate industries are now changed to smart and automated execution using blockchain for fund transfer, property transactions, etc., and preserve the details in a blockchain ledger to certify digital ownership. Thus, there is no need for trusted intermediary.

Frictionless and Secure Transactions: Blockchain having an enhanced security protocol and a superior way for transactions without high waiting time. From listing for sales to auctions for settlement clearances, a blockchain built a frictionless transaction.

Due to enhanced protocols, client information is not able to unveil by cybercriminals. It ensures the security of personal information stored in the digital system.

Global Access - Blockchain makes an international deal using cryptocurrency as a medium, for buying and selling property globally.

Streamlined - Blockchain embedded smart contracts is ultimately faster than web 2.0.

Higher Security - Due to decentralization it avoids loss of data, hacking, server crash. It ensures customers that their information or data cannot be view, sold or bought.

Lower Overhead - Blockchain implementation over insurance is inevitably a trusted system, error-free, faster and eventually generates a lower the premium amount for policyholders. This system is projected that it can able to reduce a $15 \%-25 \%$ current fraud rate in insurance. Besides, it also saves $\$ 10$ billion outlays that are incurred by insurance fraud in the industry.

Legal transaction: Entire transactions are legal. No chance for mutability and illegal transactions.

Secure cash management, clearing settlements, and client account reconciliation.

It affords a great potential environment to interchange assets without the interference of intermediaries. It also enhances online assets authenticity and tractability by storing data in a permanent ledger. 
etc. ii) Voter registration eligible candidates must register using user interface with valid ID. The unique wallet is provided for all successfully registered candidates so that no one can able to found which wallet matches to a particular individual. iii) Voters Transaction: if voter registers their votes in the corresponding ballot, then district smart contract is triggered and check for consensus attainment. If consensus is attained, formerly the corresponding vote is appended to the blockchain. Every single vote transaction on blockchain clamps information about for whom voted for and location of the vote. Each voter receives the transaction ID for a registered vote for the verification process. iv) Vote Tallying: Each locations ballots smart contract is triggered to tally the registered votes of their own. The election result about a particular ballot is displayed by a smart contract. v) Vote Verification: voters can able to verify their vote via election commission by providing transaction ID information with corresponding PIN [28]. Broncovote (voting system) is developed using the ethereum web 3 frameworks. By using metamask and web3, it avoids the need for users to partially or full download ethereum development platform into their local machines. Broncovote system consists of i) Administrator authority to build smart contracts for voting. ii) Voters - persons who are registers in a system for voting with their valid ID's. iii) Creator has the permission to create ballots [29]. E-voting system is build using smart contract-based permissioned blockchain to facilitate liquid democracy using Proof-of-Authority (PoA) consensus mechanism [28]. Here, validators validate transactions and create blocks using PoA. The validation process is automated and does not require human interaction to monitor the system. First blockchain technology for voting is introduced by the Danish political party in 2014. Digitally secured E-voting platform namely Followmyvote, is proposed for legitimate e-voting process.

Table 2:Real-Time Implementation of blockchain in Business Sector

\begin{tabular}{|c|}
\hline Real-Time Implementation of blockchain in Business Sector \\
\hline $\begin{array}{l}\text { Abra creates a platform where anyone can invest in cryptocurrency via mobile app. The digital crypto } \\
\text { wallet assets are securely stored and maintained [24]. }\end{array}$ \\
\hline $\begin{array}{l}\text { Barclays Bank is aware of the impact of blockchain. They establish a blockchain-enabled environment for } \\
\text { faster transaction, faster authentication, prevents fraudulent via transparency and hold data integrity } \\
\text { throughout the entire system [25]. }\end{array}$ \\
\hline $\begin{array}{l}\text { Propyis a blockchainusecase used in the real estate industry for a property transaction. Via Propy anyone } \\
\text { can securely buy, rent and sell a property. Due to transparency no way for fraudulent occurrence [26]. }\end{array}$ \\
\hline $\begin{array}{l}\text { Exonumblockchain platform offers a decentralized platform for secure storing of digital assets and } \\
\text { properties under complete user control [27]. }\end{array}$ \\
\hline
\end{tabular}

\subsubsection{Taxation}

One of the biggest potential contributions of blockchain for PWC (Public Works Commission) is taxation. A huge gain of using blockchain in taxation is cutting administrative costs for Value-Added Tax (VAT), stamp duties, withholding tax transactions. For uniform taxation countries like India via GST, blockchain helps to an end-to-end collection of tax automatically using smart contracts. In most of the developed countries, payroll tax payments from an employer are digitalized. Blockchain implementation reduces the act of intermediates such as maintaining of individual records, calculating the amount of tax, transferring and payment of social security taxes from employee's salary. But, a smart contract automatically calculates and pays the net amount of tax if once employer gross amount salary is credited. It resultant a fast and cost-efficient payroll tax system. Multinational transaction of goods, social security taxes, etc., can use blockchain for audit review of local files, records, calculating and executing transactions automatically. It reduces the intrinsic problem of tax transfer pricing (in Taxation Transfer pricing, knowledge is lacked about involvement of unrelated parties in the particular transaction) eg. Buying medicines, clothes, groceries, etc [30]. Various benefits for using blockchain for taxation are: It significant drops the transaction cost which should to pay for several participating intermediates, and also prevents the outset fraud and tax evasion risk. 


\subsubsection{Legal and Law enforcement:}

Intelligent based coding is integrated for a legal interface. Two possibility forms (legal side and user side) should be considered while designing the contract. Uniform contract designs are created for civil practice to execute the rules, governing practices and for maintaining legal proceedings.

Vernon Bogdanor upholds constitution rules into two ways: Foremost rule is a selection of law and legal enforcement rules that regulates and governs the government, that should be exemplified as a document like the French Fifth Republic constitution (1958) and American constitution of 1787. In second phase, rules are framed that must satisfy the bounding group of people. Most important rules for the constitution are the distribution of power among government organization as the legislative, judiciary, executive, state and individuals.

A constitutional Legal interface format exemplified by Bogdanor represents a framework that shapes ideas and concepts of rules. Alexander Galloway paraphrases the existence of a legal interface in the constitution after decentralization. Here, the legal interface not merely technical or legal but also political. Besides, a legal political interface could be organized from the paradoxical intermixing of centralized and decentralized technologies that must shape the jurisdiction authority and realm countrywide sovereignty. Traditional coding paves the way for the paying process as per contracts or by breaking the contract. Sometimes breaking of rules is not able to be found. Though, the payment process is automated by smart contract there is not having a chance for breaking of law enforcement. An essential elucidation of a smart contract could effectively proclaim the pre- defined governing code as a law. It should be self-contained, self-triggered, selfperformed, self-administrator, and self- enforced. Unlike a blockchain as a record-keeping, block- chain for legal and law enforcement must add extra feature dimensions of actions and complexity. This ensures that the system might require more processing power for maintaining and mining process. It leads to higher cost and higher energy requirements. Smart contracts Code as law ideology, have a chance of making vulnerabilities and several practical challenges. These problems may be reduced by the integration of first-generation smart lawyers using trial and error practice [31]. Legal and law enforcement must maintain i) consistency preserving the sequence of evidence ii) immutability of law and legal enforcement records iii) inhibiting the use of fake identities and easily track criminals existence across various borders iv) ensure secure intelligent information sharing and $\mathrm{v}$ ) easily track terrorism information by also protecting privacy of defense data.

The problems faced by traditional system and problems rectified by blockchain system in Government and public applications is shown in table 3, and Real-Time implementation of blockchain in Government and public applications is represented in Table 4.

\subsection{Education}

Blockchain technology avoids the traditional way of paper based certificate system and it also evades the need for the education system of government to validate the credentials of students. The education network is standardized and automated via the decentralized distributed autonomous network. Self-Sovereign IDs significantly reduces data management cost of educational organizations. It acts as a sophisticated way for reliable tracking of intellectual educational possessions.

Student information might consider as worthless which actually a pretty commodity among cybercriminals. Cyber criminals targets and steal student records that are used for creating fake identities and certificates to be sold by offenders. The aforementioned problems are rectified by using blockchains in way of storing education records, certificates, and student identities. Blockchain maintained individual student records from kindergarten to university. It ensures students security and privacy of records [34].

Basically, Blockchain is used in education for Permanent secure storing system for certificates

- Easy verification of Multiple-step accreditations.

- Automatically transfer of credit and predicts appropriate student for recognition based on credits.

- Blockchain act as a lifelong passport for student attainment in education.

- Receiving payments for fee

- Providing funds for the student in terms of vouchers

- Blockchain act as verification of sovereign IDs for the student identification system 
Table 3: Problem faced by traditional system and rectified by Blockchain system in Government Sector

Voters' identities are held by the centralized system. It has a chance for creating fake identities, can remove authorized persons. No authorized way for storing voter's identities.

The traditional voting system uses ballot papers or EVM machines (Electronic Voting Machine) is a centralized controlled system hence, it may have a chance for mutable as a favor.
Voters identities are stored in blockchain assure security and immutability of identities. It ensures entire systems are completely anonymous.

Decentralization among voting system ensures no chance for mutability which restricts to make favour of voting manually.
ํㅗㅁ

Registered votes cannot be assured for secrecy and mutability. There is a chance to register fake manually via ballot papers.
Registered votes are completely anonymous and publicly available. Anyone can cross-check voter identity and registered vote. However, the vote to the party is masked behind a randomly generated encryption key. It ensures the privacy and security of voters.

Traditionally, all certificates, audits, legal data, All documents in blockchain are stored digitally. evidence, and reports are in paper format. Blockchain simplified entire court procedures like Consequently, verification of all documents took verification of reports, documents, certificates, evidence, bound-less time to verify entire documents even for a audit process automatically via smart contracts.

¿ small issue.

\& Paper-based criminal records have a chance to make Storing of criminal records on blockchain makes a mutable and tamper the records. Secure storing of secured storing, immutable and tamper-proof of criminal documents causes a burdensome process for documents. It makes case-solving easier with less legal actives.

burdensome.

Some ambit of the law was not amended for its tedious paperwork. These law practices are amended as word-of-mouth informally.

Using blockchain tedious paper works can be prepared, organized, and verified fastly via smart contracts and that prevents the informal word-of-mouth contracts.

Even though payrolls are digitalized, there is some significant flaw due to the involvement of laws in several government institutions. Each institution has its own rules and registers. It affects the entire payroll process.

Using of blockchain for payroll payments process that inhibits mediators like the person involved for tax calculation, tax collection, transferring of tax, social security payments, and tax verification in various government institution sections.

Variation of taxes due to the involvement of Tax price is fixed mediators

A cross-border transaction is a cumbersome task.

ᄃ Various countries have different types of taxes with a

을 different mode of payment (dollar, rupees, pounds,

All tax payments are accomplished by smart contracts automatically via cryptocurrency. So, it evades difficulties arises during a cross-border transaction. etc.). Yet, there is a nope seamless system for tax collection.

Table 4:Real-Time Implementation of blockchain for Government and for public sector applicants

Real-Time Implementation of blockchain for Government and for public sector applicants

Blockchain grounded voting system that affords whole transparency in the election process. It guarantees election is piloted insecure way and ensure $100 \%$ accuracy of handling election [32]. 
Factom Software:Factom is software, developed for protecting government, non-profit systems, and critical

commercial data from data theft. The software guarantees data integrity, honesty, trust, and immutability [33].

Blockchain must perform,

1) Maintaining Student records and credentials Nowadays students learn a lot of things from inside and outside of classrooms. They learn different sources all over the lifetime. Academic records are not able to encapsulate all processes. But, blockchain offers a lifelong secure collection of all competency indicators such as academic records includes certificates, badges, citations other records, etc, which are automatically verifiable, updatable and provides immutability.

2) Partnership platform

New York City College is one of few universities that introduced payment of a fee by way of Bitcoin. Blockchain based smart contracts enable distributed and encrypted digital transactions between two parties. It ensures the secure, cost-effective, speed and transparency.

3) Copyright and Digital rights protection Blockchain is capable to share, manage and protect digital assets or contents which helps higher-end Deans, Principals, Administrators, Management, faculty members and researchers to create intellectual property and share it among different professionals but, still putting control the way of using the property on hand. Professors or any faculty members are rewarded for their teaching materials based on actual use, and for research papers based on citations.

4) Library Management Libchain Lib chains vision embraces the creation of a library ecosystem environment that shares the book database to every customer irrespective of their registered library, can reserve, request and purchase books from any partaker library or from a customer who hired a book from any of cooperating library [35].

The problems faced by traditional system and problems rectified by blockchain system in Education is shown in table 5, and Real-Time implementation of blockchain in Education is represented in Table 6.

\subsection{Healthcare}

Blockchain application in Electronic health information exchange (EHR) :

Aim of HIE is providing efficient and secure delivery of healthcare data beyond institutional and geographical boundaries. A lot of aspects to be considered for sharing data are infrastructure, security, and interoperability. All medical documents such as lab reports, physicians, medicines, records, etc, are encrypted by a private key and only a few traditional centralized paper-based transformation of drugs supply chain system. It is overcome using blockchain via open, transparency and tracking supply chain system. Using distributed ledger all participants like raw material supplier, manufacturer, transporter, retail shopper, government, retail shops, and a patient can easily track the drugs' cur- rent status quo and location using timestamps. The permanent record has been maintained from the raw material col- lection for drug preparation to in the pharmacy as a drug. The records entered are immutable, decentralized, permanent and distributed. This turns over easy tracking of malicious intent nodes in the supply chain which controls the occurrence of vulnerability due to fraud or human errors. Counterfeit drugs are greatly reduced by maintaining logs at each level of the supply chain [2] [42].

Blockchain in Claim and Billing Management: One of the main challenges in claiming and billing process is preventing fraudulent billing. Some of the most common frauds in healthcare are claiming bill for providing unnecessary services unrelated to patients medical condition, overcharging of actual services, claiming for non-performed services, claiming uncovered medical services as a service. There are many mediators involved to add, verify and adjudicate the billing and claim procedure which make a trustless administrative system. But, Blockchain reduces mediators by executing smart contracts automatically from admission time of patient to or discharge date, and achieve transparency in billing and claim process [42].

\section{Blockchain for Personal Health Record (PHR) and National Health Record (NHR):}

Due to recent abundance growth of artificial intelligence (AI) entrenched in IoT devices in the healthcare system more number of wearable devices such as sensors has been developed to predict human's health condition. It maintains the personal data management system about a human's health condition called personal 
health records (PHR). Real-time artificial intelligence (AI) healthcare analytic sensors monitor and fed information to related participants including patients, doctors, physicians, pharmacists, and relatives, etc. Decentralized distributed applications (Dapps) are developed using ethereumblockchain is under development which enables easy participation of patients, doctors, pharmacist and physicians in telemedicine without mediators.

Table 5: Problem faced by traditional system and rectified by Blockchain system in Education sector

\section{Problems Faced by Traditional System}

Secure File Storage: Without a doubt, there is a responsibility of schools to ensure confidentiality and to protect the student information. But, cybercriminals extracts student information easily from the cloud or system.

Presently, all students' academic credentials (certificates, records) are in papers. Traditional technologies have problems like missing certificates, impairment, vandalization by offenders, etc. Moreover, certificates are not able to reproduce frequently as needed. data centers and make an attack to crash the entire

\section{Problems rectified by Blockchain System}

Blockchain paves the way for secure storage of student information and ensures immutability, tamper-proof of student's data. At hand, there is no possible way to extract student information and made an attack over it. The system should withstand attacks engendered by cybercriminals.

Blockchain facilitates maintaining of every student certificates from KG to Degree, all other certificates from co-curricular activities like training, industrial visits, additional course certificates and also extra-curricular certificates achieve from sports, music, etc. can be stored digitally. DLT (Distributed Ledger Technology) streamlined the verification process and reduce the earning of fraudulent certificates for credits. For its digitalization, certificates are reproducing anywhere as needed and there is not have a chance for missing of certificates, tampering, vandalization, etc.

Most academic institutions collecting a handful of Authorship allows authors to publish their work on a research papers from all persons inside the institution. blockchain-based platform. Association collects and It structured and publishes a handful of research papers as an Oligopoly. If particular persons contribute papers but not a part of institution association then a specific person's work is unnoticed. These blocks the way of attaining credits for their own work.

publishes a handful of papers from blockchain-platform. It paves a way for authors to attain credits. Readers can purchase the articles, papers or books from blockchain platform via Authorship Tokens (ATS), and authors get $90 \%$ of royalties in the ATS system. Here, the author has a right to apply for copyright, freedom to distribute articles.

Reputation of students: Generally, criminal activities history or reputation about the student is not known. During recruitment, HR managers do not have any By storing information on blockchain verification of student or interviewee takes less time and the hiring process moves forward quickly. verifying certificates and background checking process, the HR manager can somehow predict interviewee. These processes are time-consuming and have a chance to recruit offenders.

This process reduces the cost and time taken for the mediators services. NHS states to the Governmentsubsidized medical and healthcare service center in the UK. Persons in the UK can utilize the service without paying the cost. The service includes consulting doctors, surgery, treatments at the hospital until getting well, pregnancy period, and ambulance service. All information is securely maintained and management via blockchain [43].

\section{Blockchains in Neuroscience:}

The modern neuroscience system seeks a new system paradigm to control devices and data over humans mental commands. These systems can interpret and read the brain activity patterns and convert the signals as commands. This process can facilitate to detect the current mental state of the person and to control external 
devices based on data signals received from their brain activity. Such a special task of reading and interpreting brain signals can be achieved via neural interface devices fortified with computing chips, sensitive sensors, and wireless communication. The equipped fortified device should be able to wear on the head. In this way, the devices can easily read and interpret the brain electricity signals, deciphered it and stored in equipment. Big data and complex algorithms in neural activities will utilize the philosophy of blockchain to store brain signals in a secure way.

Table 6:Real-Time Implementation of blockchain in Education

\section{Real-Time Implementation of blockchain in Education}

- A student lead non-profit organization $501 \mathrm{c3}$ "Blockchain at Berkeley" offers blockchain-related research help to start local businesses, blockchain-related education, and consulting. Elsewhere "Cornell Blockchain" is a mission started to develop, support and consult emerging blockchain projects to create innovators communities [36].

- MIT is a front-runner in blockchain-based credentials joined with Learning Machine organization has developed an "open standard for verifiable digital records".

- "Student-owned digital credentials" is a blockchain product that is delivered in 2018 by New Mexico Community College for maintaining students' information [37].

- Blockcerts is an open standard blockchain credential [38]

- RecordKeeper Company creates academic certificates and secures records using blockchain.

- Sony and IBM partnered together to create blockchain-based data storage for education [39].

- Anblockchain-based chatbot app developed by Opet Foundation helps students for preparing test by recommends resources and answering the questions whereas it keeps track of the progress of the student in blockchain[40].

- "Airbnb" is a blockchain grounded file storing platform. In Airbnb, Filecoin has a great potential for storing educational institution's students' information in a safe way [41].

Eg. One of the firms conforms and registered in Geneva in 2017 that they will be using blockchain technology for Neurogress. The primary focus of the firm is to building neural-control systems, which enables users to control smart applicants, AR/VR (augmented reality/virtual reality) devices, drones, and robotic arms with their own thoughts. Machine learning techniques are used for Neurogress control system to improve its brain-reading accuracy, for that requires retaining $90 \%$ of brain-data are needed to train the AI ingrained in the system. According to the firms white paper, big data of neural activity for the human brain project is needed 1 exabyte ( $1 \mathrm{~EB}=1$ billion $\mathrm{GB}$ ) of storage memory. At this point, the firm intends to use blockchain to preserve data storage security and privacy. It guarantees the resistance of data against hackers and proposes a neuro system to be open and transparent for potential users working in Neurogress platform services. Breaches are easily traceable and the entire system assures ecurity and confidentiality of personal data [2].

Blockchain for Point-of-Care Genomics: According to TimiInc, the worth of patients' medical record data is estimated as $\$ 7,000$ per year. Most of the medical companies developing blockchain-based services target the potential patients to buy and sell their own personal electronic health records (PHRs), wellness routine monitored details gathered by wearable sensors and personal genome data. Currently, many companies have been proposing storing DNA sequencing for particular timespan. The 23 andMe company was founded in 2006 that has most prolific and propose a direct communication between DNA testing services and participated peers. However, the major issue in the healthcare industry is privacy. 23andMe Company proclaimed that they have sold \$300 million stakes to pharmaceutical giant Glax-oSmithKline by handling access of approximately 5 million customer's exome data efficiently. The Blockchainstartups are now developing projects in medical and healthcare claims to propose a solution for data ownership. For example, a blockchain-based startup named Nebula Genomics provides a free genome-sequence to improve their genetic stock market business strategy. If users have their own sequenced genome, they can charge a fee in tokens from a person who needs to access the data. Genemes.io is another genome company that allows humans to store their genome sequence and consequently provide access grant for the owner. The aim and idea of the company are to prevent the getting genome information into wrong hands and also provide an opportunity to owner to sell their genomics data bit by bit if they wish [44]. 
The problems faced by traditional system and problems rectified by blockchain system in Medical and Healthcare is shown in table 7, and Real-Time implementation of blockchain in Medical and Healthcare is represented in Table 8.

Table 7: Problem faced by traditional system and rectified by Blockchain system in Healthcare

Problems Faced by Traditional System

No origin of medicines, ownership details and its components are identified.

At present, At the shipment of medical drugs, shipment details are tracked from information given by the shipping sectors. Crucial issues are raised to compromise fraudulent during shipment. Any compromise can affect the well-being of the patient and even leads to bereavement.

The traditional system has a range of issues including data integrity, data confidentiality, data sharing, data privacy, patient registering and record-keeping and so on. At this juncture, cyber offenders can have a chance to extract and tamper the data for their favor. No secure storage of medical documents.

\section{Problems rectified by Blockchain System}

Origin of raw materials, ownerships, manufacturer of pharmaceuticals at each stage are easily tracked and identified. It avoids the stealing and forging of pharmaceutical goods.

Medical Fraud Detection: To reduce the occurrence of fraudulent complexity, a blockchain-based supply chain platform provides drug transparency and traceability throughout shipment.
No data integrity preserved in conventional Electronic Health Record (EHR)

Neuroscience research in conventional system arises a crucial computational complexity owing to un-secure storing of data, able to tamper, and inefficiency to execute automation of integrated system consists of several sensors, integrated chips, and wireless communication system.
Clinical Research: Blockchain platform along with $\mathrm{Al}$ and $M L$ fortifies a framework for any information collaboration by manipulating medical records that grandly assures data integrity, data confidentiality, secure data sharing, and data privacy. Offenders are impotent to access data on a blockchain.

Data integrity of EHR is well-preserved via the blockchain platform. No need for human intercession.

Blockchain along with $\mathrm{Al}$ and $\mathrm{ML}$ automates incorporated system comprises of several sensors, integrated chips, and wireless communication system. The digitalization of neuro activity of the brain requires some prevailing medium to store brain activity. An anticipated system can handle big data and complex algorithms intricate in neural activities will utilize the philosophy of blockchain to store brain signals and securely impose a communications system.

The current system has more manual mediators for storing, billing, verifying, etc. grounds high cost.
Cost efficient: A smart contract automates billing, storing and verifying of healthcare and medical records which consume less cost.

\subsection{Internet of Things (IoT)}

Recently, the Internet of Things (IoT) emerges as the most promising field in information and communication technologies (ICT). IoT is proposed to incorporate several things (smart objects) via the internet which provides various smart services for users. It includes smart homes, smart grids, Radio-Frequency Identification (RFID) technology (ISO, 2013), e-health, and Maritime Industry, etc. Blockchain has the potential to improve the IoT sector. In E-Business sector, blockchain and smart contract execute a transaction for a smart property [52]. In this prototype, a decentralized transaction is espoused by Distributed Autonomous Corporations (DAC).

The trading sector uses DAC to autonomous cars, automated fuel exchange sensor data and to attain digital currency for a trade without the involvement of any third-parties mediators. Several industries are working to 
explore the potential applications of IoT entrenched with Blockchain to improve security, privacy, efficiency, reliability, transparency, and automation [53]. Some real-world applications of IoT with Blockchain are,

Table 8: Real-Time Implementation of blockchain in Healthcare

\section{Real-Time Implementation of blockchain in Healthcare}

- MedRec 2.0With the help of the Robert Wood Johnson Foundation and the MIT Media Lab, the EthereumBlockchain is being created for improved access to medical records. MedRec 2.0 is now being trialed and hosted at Israel Deaconess Medical Hub. The source code is freely available via open-source option. [45].

- Digital Treasury Corporation (DTCO) and Taipei Medical University jointly developed PhrOS for maintaining pateint's record. The goal of PhrOS is to promote patient record transparency by putting patient records from diverse medical institutes on a Blockchain. The project is now complete and available on the phrOS website. [46].

- Coral's Health discovery and research organization incorporate Blockchain to maintain lab data and treatments, whereas, it also aim to use blockchain technology to speed up patient care, automates administrative processes, and incorporates smart contracts between patients and doctors. [47].

- FarmaTrust is a product developed by ICO with the goal of eliminating counterfeit pharmaceuticals. It keeps track of manufacturer and regulatory compliance aids that adhere to government regulations, and inventory can be traced and tracked easily using Blockchain. Anyone may readily track any changes made to a product using supply chain visibility management. [48].

- $\quad$ MTBC :By altering the Application Program Interface, MTBC wants to improve the traditional Electronic Health Record system (EHR) system. Blockchain technology enables the full controls of medical records are in the hands of patients. A patient can transfer his or her own reports from one doctor to another via MTBC. The Hyperledgerblockchain platform is currently accessible. The MTBC project can be found on the MTBC website. [49].

- Hashed Health product intends to make a transparency of medical sector professional credentials. It enables credentials are easily tracked and verified by authorized members of the chain. It streamlines the unalterable history of medical professional's education and career [50].

- "Change Healthcare" streamlined the regulators and security involved in a healthcare transaction. The product is available in the Change Healthcare website. It simplifies the health system's remittances, claims, minimizes denials, evades high and down payments, manage the business operation and manages daily revenue cycles effectively using blockchain. As stated by the website, $92 \%$ of topmost US health centres plans to utilize their services [51].

Some other real-time applications of blockchain in healthcare are [51]

- Factom - secure storing of Digital Health Records.

- MedicalChain, Simplyvital Health, Robomed - to maintain the integrity of patient records from an outside source.

- Guardtime - To maintain data privacy of cyber systems including Healthcare.

\subsubsection{Automotive Industry}

Automotive Industries are now developing a fully automated system using IoT empowered sensors. Decentralized IoT enabled sensor network and devices provides an admirable platform to exchange crucial information quickly and easily among multiple users. The IoT grounded automotive industry is one of the interesting fields to integrate blockchain technology which enables of any third-party mediators.

EgNetObjex.NetObjex established a smart parking system using IoT entrenched with blockchain. It easily enables the process of detecting a vacant space in parking and automates billing directly via crypto wallets. NetObjex collaborates with real-time vehicle detection and parking sensor firm PNI to automatically detect the parking area. IoT sensors manage parking space and crypto wallet is maintained by blockchain. 


\subsubsection{Smart Cities}

Cities that are incorporated with Information and Communication Technologies (ICT) intents to enrich or enhance the performance and quality of urban services like transportation, resource sharing, energy, and utilities in order to reduce wastage, cost and resource consumption are called Smart Cities. Information and Communication Technologies (ICT) along with IoT and blockchain technology enhances better utilization prospects of sharing of resources among smart cities. IoT sensors gather various information like temperature, the existence of disaster, population, shared economy rate, current market rate, sharing resources, etc, and are stored on a blockchain. It ensures immutability, proper as- set utilization (data), accountability and transparency among the entire network.

\subsubsection{Smart Homes}

Recently, Smart IoT facilitated devices play a vital role in our day-to-day lives. IoT entrenched smart devices enable a home security system can be organized, controlled and managed from the smartphones. Owing to a lack of information ownership and security standards by centralized IoT devices, blockchain is incorporated in IoT to prevent the centralized network and solves the security issues by granting permission.

Eg.Telstra. Telstra is an Australian telecommunication, and media company which provides smart home applications. One of the applications of Telstra is advancing biometric security using blockchain. It ensures nobody can able to modify or manipulate the biometric data, captured by smart devices. Sensitive biometric data such as fingerprint, facial recognition, voice recognition, and iris scan, etc., are stored in the blockchain in order to improve security by decentralization immutable and permission granting aspects.

\subsubsection{Supply Chain and Logistics:}

An international supply chain network involves many parties such as raw material suppliers, brokers, manufacturers, retailers, suppliers, etc. Due to the involvement of numerous stakeholders tracking and delivery of a product is the biggest challenge. Nowadays some companies are working on IoT entrenched with blockchain to make reliable and transparency in tracing of the entire shipment process without any impediments. IoT sensors namely vehicle information, GPS, connected devices, motion sensors anticipate the shipment status. The anticipated sensors information is stowed in the blockchain.

Eg. Golden State Foods (GSF) is an eminent manufacturer and diversified food supplier which serving more than 125,000 restaurants with the aim of producing and delivering high-quality food products. Currently, GSF and IBM jointly working together to optimize global GSF business processes using IoT and blockchain. An IoT sensor gathers shipment and business deal information and stores on a blockchain. It allows GSF to ensure improving accountability, immutable, secure, and transparency.

\subsubsection{Sharing Economy}

The sharing economy allows the sharing of goods to earn revenues seamlessly. Now, sharing economy perception is adopted worldwide. Eg. Slock.it firm facilitates the sharing of IoT objects using blockchain to enable the economy of things. They are developing a strategic plan known as Universal Sharing Network (USN) which is used to connecting things for the secure online market. Through USN, any things or objects can be securely sold, shared and rented without mediators. It allows third-parties manufacturers to shipboard any products to USN without seeking permission. Blockchain-based smart contracts ensure data security, privacy, and transparency by governing access to information.

\subsubsection{Agriculture}

Tracking of food products in the supply chain requires transparency to ensure security and trustworthy. IoT entrenched with blockchain has a great potential to restructure the entire food production industry from farming field to grocery to home. By mounting smart IoT sensors in farming fields and in suppliers domicile it directly collected information to the blockchain.

Eg: Pavo is a food mart that uses blockchain and IoT to enhance the farming sector. The IoT sensors installed in agricultural fields gather farming information like crops stages, maturing of the crop, etc., and informed to retailers, distributors, and consumers about the particular crop. It prevents the waiting time of farmers by preselling all crops via a smart forward contract for payments after harvest. The problems faced by 
traditional system and problems rectified by blockchain system in IoT is shown in table 10, and Real-Time implementation of blockchain in IoT is rep- resented in Table 11.

Table 9: Problem faced by traditional system and rectified by Blockchain system in IoT

\begin{tabular}{|c|c|c|}
\hline Problem & Problems Faced by Traditional System & Problems rectified by Blockchain System \\
\hline \multirow{4}{*}{ 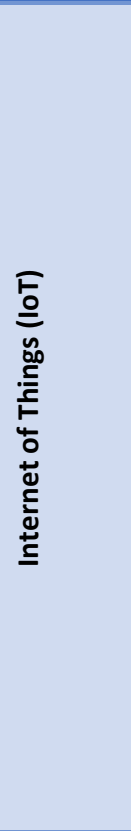 } & $\begin{array}{l}\text { Currently, no proper way for payments. } \\
\text { Payments take place via manually mutable } \\
\text { digital gadgets or paper-based documents. It } \\
\text { pays the way to forge in payments. }\end{array}$ & $\begin{array}{l}\text { Blockchain grounded digitalized system creates a } \\
\text { straightforward and honest payment system. Owing to } \\
\text { crypto enabled hardware and immutable property no } \\
\text { one can forge in custom payments. The system has a } \\
\text { significant level of security in custom payments. }\end{array}$ \\
\hline & $\begin{array}{l}\text { Deployment and organization of loT devices are } \\
\text { tedious processes owing to verify } \\
\text { authentication, identification of each device } \\
\text { accompanied by seamless secure data transfer. }\end{array}$ & $\begin{array}{l}\text { Identification and authentication of entire loT devices are } \\
\text { verified rapidly and organized a whole as perfect with } \\
\text { seamless secure data transfer. }\end{array}$ \\
\hline & $\begin{array}{l}\text { No healthy transparency throughout the cargo } \\
\text { shipment process. Owing to less transparency, } \\
\text { tracking of goods is problematic. So, there is a } \\
\text { possibility to make a fraudulent of goods during } \\
\text { shipment. }\end{array}$ & $\begin{array}{l}\text { Blockchain platform incorporated with crypto locks, } \\
\text { sensors, soft, and hardware can create an immutable, } \\
\text { transparent, secure, efficient and reliable system. Smart } \\
\text { contracts automate the tracking process to track goods } \\
\text { accurate location, other details like person handle cargo } \\
\text { at current situations, transportation information. This } \\
\text { automates the healthy shipment of goods. Eg } \\
\text { Machineries, devices, etc. }\end{array}$ \\
\hline & $\begin{array}{l}\text { Single point of failure in a centralized system } \\
\text { can cause blocking of the whole system's } \\
\text { ecosystem }\end{array}$ & $\begin{array}{l}\text { DLT eliminates the occurrence of obstructive arises in } \\
\text { the system owing to a single point of failure. }\end{array}$ \\
\hline
\end{tabular}

Table 10:Real-Time implementation Blockchain In IoT

\section{Real-Time implementation Blockchain In loT}

Maerskis a transport company established for efficient tracking of cargo movement. Due to integrity, security, and transparency of the system, transport ports or firms will have healthier control over goods. It restricts illegal transport [44].

NetObjex -NetObjex has developed a consistent, decentralized communication system for loT devices. The blockchain-enabled loToken offered by the company provides a secure digital platform for interacting and communicating with smart devices in the same network ecosystem [54].

Helium's based blockchain is used to strengthen wireless Internet Infrastructure using radio technology that drastically reduces the power required to run smart machines [54].

ArcTouch is a firm, focus to develop a blockchain grounded software for various connecting loT smart devices including smart TVs, wearables [54].

Firm "HYPR" uses blockchain to securely connect ATM's, homes, and cars. HYPR focuses on build blockchain to store biometric logins namely iris, palm, facial data, and voice protocols of participants to enable loT devices [54]. 
Provenance: Provenance fuels the entire supply chain including producers, shoppers, retailers, and shareholders. It creates a fully transparent environment, wherever any participated peers can easily track and identify product origin, and other vital information [55].

"Slock.it" uses blockchain technology to share loT equipment and items. They intend to create a Universal Sharing Network (USN) that paves the way to share, sell or lease loT devices without intermediates.

\subsection{Cyber security}

Cyber-security is a process of protecting electronic systems like mobile devices, computers, servers, networks, data, and electronic systems from malevolent attacks. It is also called as Electronic Information Security (EIS) and Information Technology Security (ITS). Generally, cybercriminals are endeavour to steal valuable data like financial data, intellectual property (IP), health records, personal identifiable information (PII), Business data, and asset information etc., and convert them as highly profitable strategy (asking compensation) in the ways like disturbing entire business operations by means of Distributed Denial of Service (DDoS) attacks or by ransomware attacks [56]. Here, blockchain is incorporated in order to overcome challenges existed in cybersecurity. Some manifestations are:

Advanced Confidentiality and Data Integrity:Blockchain engrained cyber devices ensure data confidentiality, integrity and access control. The complete encryption process of the blockchain system ensures cyber devices data during transit or realm in the system are not able to access by any wrong person or organization.

Improved PKI: Public Key Infrastructure (PKI) retains the secure communicating system, emails, applications, websites, etc. However, these systems rely on third-party credential consultants to broadcast, revoke and store key pairs. These specific credential consultants can become an easy target for cybercriminals to penetrate and retrieving encrypted communications via spoof identifies. But, in the case of using blockchain for publishing key pairs, there is no possibility of generating spoof identity key pairs that restrict the entry of hackers [57].

Protected Edge Computing with Authentication: Edge computing enhances productivity, power usage, and efficiency if IT industry but, facing security challenges in terms of authentication, record management, access management, and data transmission. Although, blockchain strengthening the aforementioned discipline and enrich edge computing.

Secured Private Messaging: A lot of organizations are looking forward to blockchain technology for securing their personal and private information exchanged via social media, messaging apps, and chats. They expect blockchain can help to generate a secure platform for messaging apps and hindering foreign attacks.

Diminished DDoS attacks: DDoS can attack network re- sources, transmitting data, servers, computers, and websites. This impels to slow down the system or even crash so that denying legitimate users services. This problem can be elucidated by implementing blockchain into a decentralized network that can shield the system from DDoS attacks.

Intact Domain Name System (DNS): Hackers or cyber- criminals can easily acquire DNS service providers of leading websites like Facebook, Twitter, Patym, and Paypal, etc. This difficulty can be removed by storing DNS entries within blockchain which resist hackers from accessing these DNS.

The problems faced by traditional system and problems rectified by blockchain system in cyber-security is shown in table 11, and Real-Time implementation of blockchain in cyber-security is represented in Table 12.

\subsection{Web community}

A very significant role of the web community is accessing the reputation of contributors. Carboni (2015) suggested a new reputation model system based on blockchain. Here, a voucher or coupon has been signed if a customer is satisfied with the service and desires to give good feedback. If a vouchers signing is favor in sense of providing good feedback for service provider then, the service provider pays an extra $3 \%$ as compensation for as a voting fee to the network in order to diminish Sybil attacks. The reputation of a service provider is deliberated based on a proportion of voting fee. A new reputation system is designed by Dennis and Owen (2015) using blockchain, which can be practically implemented to multiple networks [62]. Specifically, in 
this system, blockchain is used to accumulate a single dimension reputation value (either 0 or 1 ) given by completed transactions [63].

For example, consider peer A is sending a file to peer B. After receiving a file, peer A sends file hash, private keys, and score to verify the identity of a file to peer B. Afterwards the miners in the system contact peer A and B to confirm eventuating of a transaction without suspicion. As reputation score is stored on a blockchain, there is no possibility to tamper the reputation score.

Table 11: Problem faced by traditional system and rectified by Blockchain system in Cyber security

\section{Problems Faced by Traditional System}

The traditional system has a chance for data tampering, stealing of sensible messages due to lack of strong encryption procedure, auditability via manually mutable digital gadgets, and less transparency.

To secure the cyber-physical system, a password is required whenever we want to access any devices in the system to ensure identification and authentication.

The entire security of the cyber-physical system depends on centralized authorized mediators. Here, probabilities are existed to compromise cyber-physical devices to attain fraudulent.

Invaders make DNS potential attacks on a centralized server to aim to gain control of entire cyber-physical system. They block the flood of traffic "from and to" of a centralized system that ultimately crashes the entire cyber-physical system.

A single point of failure can attain by invaders by way of easily compromise the cyber-physical nodes that ascend DDOS attack. Original data are lost which makes crash an entire cyber system operation.

\section{Problems rectified by Blockchain System}

Blockchain platform potentially integrated on a cyberphysical system can prevent fraudulent, enhance security, detect and prevent tamper over data based on its aspects of unbreakable data encryption, immutable digital data storage enhance auditability, transparency, and operational resilience.

Blockchain incorporated cyber-physical devices that are proficiently identified and authenticate without the necessity of a password.

Due to blockchain aspects, no necessity of mediators to handle the cyber-physical system. The security of cyberphysical system is not able to compromise by human intervention. Thereby, it prevents potential attack vector.

Cyber-physical systems integrated with blockchain would make DNS potential attacks as, much harder to break security, more expensive and consume more time to accomplish the attack. It leads to a big challenge for invaders to achieve attacks. Outspokenly, invaders currently not have the ability to achieve outbreaks.

No single point of failure is occurred by DLT technology. DLT solves the attacks with Byzantine General's problem technique over false consensus.

Table 12:Real Time Implementation of blockchain in Cyber security

\section{Real Time Implementation of blockchain in Cyber security}

GuardTime is a blockchain project for cybersecurity. The firm intends to make use of its own KSI technology to protect user's data and is specifically designed for enterprise solutions [58].

Remme-this project intent to protect both users' and firm's data, records, documents form cyber-attacks [59].

Blockverify uses blockchain solution to identify counterfeits. Its role is to recognize counterfeits and provides a non-duplicated environment. Some eminent use cases include electronics, pharmaceuticals, and electronics [60]. 
PeerNovablockchain technology enhances financial world operation by consuming the best aspects of Blockchain, BigData and Cloud technologies. The technology aims to secure financial data, enhance audit, compliance, and reconciliation. This also allows them to tackle their audit, compliance, and reconciliation. Three main characteristics of PeerNovaBlockchain technology are redaction, immutability and logic playback [61].

\subsection{Human-Rights and Contribution}

Insinuations of blockchain for human rights are focused to solve miscellaneous challenges. Foremost distillate challenges in human-rights contributions overwhelmed by means of blockchain are: to inhibits slave labor by preserve labor details and claims, thwarting human trafficking, perceive claim realms, tracking funds transaction, track initiatives which raise funds, track individuals or troops violates rights.

Free-speech right Blockchain is used to preserve and secure the internet infrastructure such as identities of devices (IDs) and DNS [63]. For example, Namecoin is used to dis- tribute DNS among users to participate in the network. It is an open-source technology that enhances security, privacy, decentralization, speed of DNS, identities and censorship resistance [64]. It assures and guarantees to protect the online free-speech rights act by making the web (WWW) to more resistive and censorship.

\subsection{Charity}

Donation via blockchain ensures accurate accountability, security for amount, for able to track the current location of donated value, track funds to reach the right person, and preserves integrity. Digital wallet cryptocurrency reduces the computational complexity overhead in donation payment processing [65]. Blockchain has potential to transform charitable trust as an honest way by enhancing transparency in aid distribution, the immutability of data for every single aid transactions, cost reduction via evade intermediates, an facilitating a new mechanism for tracking and monitoring aids at each step from donor to recipient, and records every action in aid distribution. For example, Alice is one of social organization incentive platform to run projects transparently and peers get paid when they achieve specific goals. A distribution of aids is reached via smart contracts if milestones are reached. Here, aid donors can easily track their donations capitalized for projects. In cross-border aid transactions, blockchain significantly reduces mediators, thus reduces inefficiently during transfer and saves costs spending for mediators.

\subsection{Royalty:}

Blockchain implementation in royalty facilitates easier publishing and distributing the inventions designed by downstream contributors like game developers, graphic designers, entertainers, and apps developers, etc [66],. and make an automatic rewards reimbursement for their inventions. Blockchain improves operational efficiency by automation using smart contracts and increases trust and transparency among industry players. Eg. Ernst Young (EY) and Microsoft together proclaim the launch of a blockchain interpretation for preserving content rights and royalties' management. The system is designed as, both the publishers and distributors have to sign up on a blockchain platform by agreeing with business terms and condition via smart contract. Via Smart contract royalty amount, copyrights, terms, and conditions are fixed previously. If a customer buys products from an online retail store, the entire transaction is recorded on a blockchain. It makes easy royalty governance [67]. At first, Imogen Heap - a British singer launched her song Tiny Human using the Ethereum-based Ujo platform for $\$ 0.60$ per download. Gaming giant Ubisoft currently experimenting blockchain technology to preserves royalty for their invented games. 


\subsection{Energy Saving and Green Energy}

A transformational change in energy systems is triggered by the advancement of the decentralized distribution of information communication technologies (ICT) and energy resources. Natural blockchain inherent features might provide a promising solution to manage and control complex decentralized energy systems and microgrids. Blockchain makes potential data transmission, storage, and communication between smart devices. Smart grid intelligent de- vices include AI entrenched advanced sensors, smart meters, smart home energy controllers, network monitoring equipment, control, and energy management systems, and develop smart monitoring systems. Blockchain could achieve asset management, flexibility services, and incorporated flexibility trading platform in energy management platform. Newly hosted solar coin digital currency is used as a reward for green energy producers (particulars for solar energy). In fact, all virtual or digital coins are attained through the mining process, solar coins are granted as rewards by the solar coin foundation until producers produce solar energy.

Table 13: Problem faced by traditional system and rectified by Blockchain system in Energy saving, Web community, Human rights, Royalty, and Philanthropy sectors

Problems Faced by Traditional System

Human mediators are required to calculate, billing and verifying consumer's energy consumption procedureand no Integrity is assured on behalf of energy meters.

\section{Problems rectified by Blockchain System}

Energy consumption is calculated and sealed with crypto tags. This system assures the integrity of energy meters with enriched transparency among energy distribution and here smart contract automates efficient billing via a digital wallet ensures integrity of energy meters.

Smart contract integrated smart meters are accurately tracking overall energy production from energy production station to energy utilization by consumers. energy production stations to energy utilization by consumers. It arises incompatibility problems between distributors, suppliers, and consumers that let to a surplus of energy wastage.

Occurrences of malicious energy trading by Due to decentralization and smart contract billing system is authorized person. protected from malicious consumption of energy and assents intelligent energy trading.

\begin{tabular}{|c|c|c|}
\hline 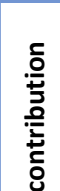 & $\begin{array}{l}\text { Free speech rights are restricted or tampered in } \\
\text { a web by some government officials or } \\
\text { authorized firms if some opinions are against } \\
\text { their favor }\end{array}$ & $\begin{array}{l}\text { "Namecoin" - a blockchain platform that distributes DNS for } \\
\text { participated users in order to secure and enhance the speed } \\
\text { of DNS, privacy, identities and censorship resistance. It } \\
\text { assures to protect the online free-speech rights act by } \\
\text { establishing a resistive and censorship web. }\end{array}$ \\
\hline 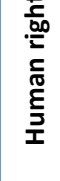 & $\begin{array}{l}\text { Current systems not having the ability to } \\
\text { preserve genuine identity or information owing } \\
\text { to hole in preserving the security of data, } \\
\text { mutable nature (have a chance to make } \\
\text { fraudulent) and easily attack by cybercriminals. }\end{array}$ & $\begin{array}{l}\text { Human rights implication intents to use blockchain to solve } \\
\text { some foremost challenges. It includes stopping slave labor, } \\
\text { hygienic and affordable housing for poor, and tracking of } \\
\text { funds from passing an amendment to at hand reach of funds } \\
\text { to beneficiaries. Blockchain can achieve the aforementioned }\end{array}$ \\
\hline
\end{tabular}




\begin{tabular}{|c|c|c|}
\hline & $\begin{array}{l}\text { Eg. Mediators can achieve fraudulent in the } \\
\text { allocation of funds, welfares distribution among } \\
\text { beneficiaries. }\end{array}$ & $\begin{array}{l}\text { challenge by preserving genuine identity and information's } \\
\text { about individuals. }\end{array}$ \\
\hline 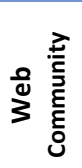 & $\begin{array}{l}\text { The current web community reputation system is } \\
\text { not efficient. }\end{array}$ & $\begin{array}{l}\text { A very crucial role of the web community is to gain a } \\
\text { reputation of contributors. Carboni (2015) proposed a } \\
\text { blockchain-based new reputation system model that creates } \\
\text { an effective web community realm. }\end{array}$ \\
\hline 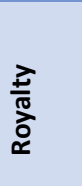 & $\begin{array}{l}\text { Fraudulent is ensued in content rights and } \\
\text { royalties during an assignment or during the } \\
\text { preserving process. Ownership, royalties of } \\
\text { patents, research works are not well- } \\
\text { maintained. }\end{array}$ & $\begin{array}{l}\text { Blockchain-based royalty system preserves content rights and } \\
\text { royalties of individuals or managements. Ownership details } \\
\text { for patents, research works or innovative ideas are well- } \\
\text { maintained. }\end{array}$ \\
\hline \multirow{2}{*}{ 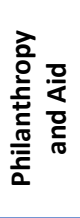 } & $\begin{array}{l}\text { Dishonest aid distribution. Not have the ability to } \\
\text { track the origin of fraudulent. }\end{array}$ & $\begin{array}{l}\text { Blockchain aspects have the potential to enhance aid } \\
\text { distribution by means of transparency, reduce } \\
\text { disintermediation cost, faster transaction, and tracking of aid } \\
\text { distribution. }\end{array}$ \\
\hline & $\begin{array}{l}\text { Aid donors do not have an idea about the } \\
\text { amount they contribute. }\end{array}$ & $\begin{array}{l}\text { Aid donors can easily track the distribution of donations and } \\
\text { view the performance of projects for what they contribute. }\end{array}$ \\
\hline
\end{tabular}

SOLshare is electricity trading blockchain platform. The objective of SOLshare is to produce electricity for distant regions of Bangladesh from local energy grid solar panels. It causes distant homes to generate electricity for their own use and also to trade electricity without relying on utilities. SOLBox is one of SOLshare's products that enable house- holds to purchase electricity as required by paying as tokens via mobile SMS. Here, blockchain enhances decentralization in trade in energy and payment, (ii) providing access to accessible energy, (iii) shifting electricity to homes as required, and (iv) providing micro-producers with a prospective source of revenue. SOLshare currently uses blockchain to develop a tokenization model to allow energy allocation transparency and grid management [68].

The problems faced by traditional system and problems rectified by blockchain system in Energy saving, Human rights contribution, Web community, Royalty, and Philanthropy and Aid is shown in table 13, and Real- Time implementation of blockchain in Energy saving, Human rights contribution, Web community, Royalty, and Philanthropy and Aid is represented in table 14 .

Table 15:Real-Time Implementation of blockchain in Charity, Web community, Energy Saving

\section{Real-Time Implementation of blockchain in Charity, Web community, Energy Saving}

Eg. The UN World Food Programme (WFP) uses blockchain to facilitate aid distribution in Jordan which transfers cash for over 1,00,000 Syrian refugees and inspect beneficiary outlay. Initially, $98 \%$ of costs ( $\$ 6$ billion) are saved annually spend on bank transfer [69]. 
Fidelity charitable is the second biggest grantmaker in a nation that in 2017 gets $\$ 70$ million endowments as cryptocurrency [70].

Brave is a blockchain grounded contemporary, safe, quick, and personal browser. The user's privacy has been preserved due to the blocking of the tracker. Similarly, the determined browser works quicker than other famous browsers, including chrome and Mozilla, owing to the blocking of ads and trackers [71].

LO3 Energy is a blockchain platform, which empowers consumers to regulate their energy consumption and production by enabling them to produce, shop, purchase, sale, and power locally $[72]$.

\section{Conclusion}

Blockchains have received much interest worldwide. In this work, we survey blockchain usage in different areas including cryptocurrency, healthcare, advertising, insurance, copyright protection, energy, and societal applications. Our paper presents a timely summary for entities with an interest in blockchain. Moreover, the discussion will motivate other researchers or enterprises try to incorporate the Blockchain technology in their own domains in order to reduce the current vulnerabilities faced by the network.

\section{References}

[1] Guardtime Telecom, "Our Business Portfolio", Retrieved from https://www.digitalinsuranceagenda.com/featured-insurtechs/guardtime-the-worlds-largestblockchain-company/, on Sep 2021.

[2] Asad Ali Siyal, Aisha Zahid Junejo, Muhammad Zawish, Kainat Ahmed, Aiman Khalil, and Georgia Soursou, "Applications of Blockchain Technology in Medicine and Healthcare: Challenges and Future perspectives", Retrieved on Oct 10, 2019 https://www.mdpi.com/2410$387 \mathrm{X} / 3 / 1 / 3,2019$

[3] NisharaNizamuddin, Haya Hasan, Khaled Salah, and Razi Iqbal, "Blockchain-Based Framework for Protecting Author Royalty of Digital Assets, Arabian Journal for Science and Engineering, volume 44,2019, (pp 3849-3866).

[4] Le Lab, "Solshare, the Startup for Sharing Solar Energy in Bangladesh", Retrieved from https://medium.com/le-lab/solshare-the-startup-for-sharing-solar-energy-in-bangladesh-en377002b21716, on September 2021.

[5] Y. Zhang and J. Wen, "An IoT electric business model based on the protocol of bitcoin", Retrieved on July 23, 2019, 18th International Conference on Intelligence in Next Generation Networks (ICIN), 2015, pp 184-191.

[6] Toshendra Kumar Sharma,"The Future of Cyber Security: Blockchain Technology", Retrieved from - https://www.blockchain-council.org/blockchain/the-future-of-cyber-security-blockchaintechnology on september 2021.

[7] Imran Bashir, "Mastering Blockchain: Distributed ledger technology decentralization, and smart contracts", Packt Publishing, 2018.

[8] BlockchainAt Berkeley, "A Student Organization focused on Blockchain Innovation via Education, Research, Design, and Consulting", Retrieved from - https://blockchain.berkeley.edu/, on september 2021.

[9] NamrataNagpal, "INTROSPECTING BLOCKCHAIN TECHNOLOGIES IN HEALTHCARE", International journal of Research and Analytical Reviews, Vol no: 6, Issue no : 1, DOI:10.13140/RG.2.2.12204.08324, March 2019. 
[10] Followmyvote, "Secure dApp Development", Retrieved from - https://followmyvote.com/ on September 2021.

[11] Blockcerts, "The Open Standard for Blockchain Credentials", Retrieved from https://www.blockcerts.org/ - on September 2021.

[12] Farmatrust, "Digitising And Innovating The Pharmaceutical And Healthcare Businesses" Retrieved from https://www.farmatrust.com/ - on September 2021.

[13] Phil Fersht, "The top 5 enterprise blockchain plat- forms you need to know about", Retrieved from2019, https://www.horsesforsources.com/top-5-blockchain-platforms_031618 on september 2021.

[14] The Linux Foundation Projects, "Hyperledger", Retrieved from - https://www.hyperledger.org/ on September 2021.

[15] PrableenBajpai, "The 10 Most Important Cryptocurrencies Other Than Bitcoin", Retrieved on Sep 21, 2019, retrieved from - https://www.investopedia.com/tech/most-important-cryptocurrenciesother-than-bitcoin/, on September 2021.

[16] BernardMarr," 35 Amazing Real World Examples of How Blockchain Is Changing Our World", Retrieved from - https://www.forbes.com/sites/bernardmarr/2018/01/22/35-amazing-real-worldexamples-of-how-blockchain-is-changing-our-world/ on September 2021.

[17] Supriya Thakur Aras, and Vrushali Kulkarni, "Blockchain and Its Applications - A Detailed Survey", International Journal of Computer Applications, volume 180, 2017,pp 2935.

[18] Christian Jaag, Christian Bachr, "Blockchain Technology and Cryptocurrencies: Opportunities for Postal Financial Services", Retrieved from - https://www.springerprofessional.de/blockchaintechnology-and-cryptocurrencies-opportunities-for-pos/12012676 on september 2021.

[19] ChainTrade, "Blockchain-Based Platform for the Physical Trade of Commodities", Retrieved from URL -, https://chaintrade.net/ on September 2021.

[20] Peters G.W, and Panayi, E., "Understanding modern banking ledgers through blockchain technologies: Future of transction processing and smart contracts on the internet of money", Banking Beyond Banks and Money. New Economic Windows. Springer, Cham., volume 17, 2016, (pp 239-278).

[21] Divya Joshi, "IBM, Amazon \& Microsoft are offering their blockchain technology as a service", Retrieved from - https://theentrepreneurfund.com/ibm-amazon-microsoft-are-offering-theirblockchain-technology-as-a-service/ on september 2021.

[22] Chromaway, "Chromia Power to Public", Retrieved from URL - https://chromaway.com/ on September 2021.

[23] Deloitte, "Blockchain Applications in Insurance", Retrieved https://www2.deloitte.com/content/dam/Deloitte/ch/Documents/innovation/ch-en-innovationdeloitte-blockchain-app-in-insurance.pdf on september 2021.

[24] ABRA, "Invest in the world"s most exciting stocks using Bitcoin", Retrieved from URL https://www.abra.com/ on September 2021.

[25] Barclays, "Blockchain Revolution in Trade Finance", Retrieved from URL https://www.barclayscorporate.com/insights/innovation/blockchain-revolution-in-trade-finance/ on September 2021.

[26] Mike Butcher, Propy, a blockchain-verified platform for selling houses, raises funding from Tim Draper, Retrieved form URL - https://techcrunch.com/2020/10/02/propy-a-blockchain-verifiedplatform-for-selling-houses-raises-funding-from-timdraper/?guccounter $=1 \&$ guce_referrer=aHR0cHM6Ly93d3cuZ29vZ2xlLmNvbS8\&guce_referrer_s ig=AQAAAGUlqZm3ms_flLh5FkYeowC31xd_AwwpQGAiLWOQXG-1gIAODJZ1TFpeQ4P8OdWgR8FMJǨnnv5uXkvzVBKa_ZeJINt4jp_JZIWVc6jbj21AQlvE5VnsaNaNrpTXVQ iI27BxnTHt_3ZbF3kPNKZKJ-PV1aVoefFIDD19gznF6bsw on September 2021.

[27] BitfuryExonum, "Build Trust into Your Organization", Retrieved from URL https://exonum.com/, on September 2021.

[28] Fridrik P, Hjalmarsson, Gunnlaugur K. Hreidarsson, Mohammad Hamdaqa and GisliHjalmtysson,"Blockchain-Based E-Voting System" Retrieved on July 23, 2019, IEEE 11th International Conference on Cloud Computing, 2019. 
[29] Gaby G. Dagher, PraneethBabuMarella, MateaMilojkovic,JordanMohler, "BroncoVote: Secure Voting System using EthereumsBlockchain", Retrieved on July 23, 2019, 4th International Conference on Information Systems Security and Privacy (ICISSP 2018)- SCITE press Publication, 2018, (pp 96-107).

[30] Deloitte, Blockchain technology and its potential in taxes", Deloitte - Blockchain implications for tax, Retrieved from URL

https://www2.deloitte.com/content/dam/Deloitte/pl/Documents/Reports/pl_Blockchaintechnology-and-its-potential-in-taxes-2017-EN.PDF on September 2021.

[31] Philip Boucher, Susana Nasciment and MihalisKritikos, "How blockchain technology could change our lives", Retrieved from URL - https://op.europa.eu/en/publication-detail//publication/9964fbfd-6141-11e7-8dc1-01aa75ed71a1 on September 2021.

[32] UzmaJafar, MohdJuzaiddin Ab Aziz and ZarinaShukur, " Blockchain for Electronic Voting System-Review and Open", MDPI, Sensors 2021, 21, 5874. DOI: https://doi.org/10.3390/s21175874.

[33] Factom software, "Data Integrity with Ease", Retrieved from URL https://www.factomprotocol.org/ on September 2021.

[34] Jasonjenkins, "Why We Added Our First Factom (FCT) Position", Retrieved from URL https://steemkr.com/cryptocurrency/@jasonjenkins/why-we-added-our-first-factom-fct-position, on September 2021.

[35] Alok Kumar Jain, "Education-Blockchain Goes to School", Retrieved from URL https://www.cognizant.com/whitepapers/blockchain-goes-to-school-codex3775.pdf on september 2021.

[36] Juan Cabello and GerritJanben and Alexander Muhle, "Libchain-Distributed library management system based on the blockchain technology", Retrieved from https://www.atositchallenge.net/wpcontent/uploads/2016/11/LibChain-Atos-IT-Challenge-2017.pdf on september 2021.

[37] Roshan Mirajkar, "BlockchainAt Mason", Retrieved from URL - https://medium.com/blockchainat-mason/blockchain-at-mason-3e4f023b7651, on September 2021.

[38] Grech, Alexander, Camilleri, Anthony F. "Blockchain in Education", Retrieved from https://www.pedocs.de/volltexte/2018/15013/pdf/Grech_Camilleri_2017_Blockchain_in_Educatio n.pdfon september 2021.

[39] Blockcerts, "The Open Standard for Blockchain Credentials", Retrieved from URL https://www.blockcerts.org/guide/ on September 2021

[40] Sam Mire, "Blockchain for Education: 3 Possible Use Cases", Retrieved from URL https:/www.disruptordaily.com/blockchain-use-cases-education/, on September 2021.

[41] Airbnb, "Airbnb Supports Out-of-School Learning by Launching Online Field Trips ", Retrieved from URL - https://news.airbnb.com/airbnb-supports-out-of-school-learning-by-launching-onlinefield-trips/ on September 2021.

[42] Reformerthoughts - UK, "Blockchain in the NHS", Retrieved from URL https://reform.uk/sites/default/files/2018-12/Blockchain\%20in\%20the\%20NHS\%20-\%20VF_1.pdf on September 2021.

[43] Dimiter V. Dimitrov, "Blockchain Applications for Healthcare Data Management, HIR-Healthcare Informatics Research", Healthcare Informatics Research, Vol no: 25(1):51, DOI: 10.4258/hir.2019.25.1.51.

[44] Maersk, "Transportation Services", Retrieved from URL - https://www.maersk.com/transportationservices, on September 2021.

[45] MedRec, "MedRec 1.0", Retrieved from URL - https://medrec.media.mit.edu/technical/ on september 2021.

[46] DTCO, "DTCO and TMU Hospital Reveal Joint Blockchain Project to Improve Patient Record Security", Retrieved from URL - https://medium.com/dtco/dtco-and-tmu-hospital-reveal-jointblockchain-project-to-improve-patient-record-security-bee98520413c, on September 2021.

[47] Sam Daley, "15 Examples of How Blockchain is Reviving Healthcare", Retrieved from URL https://builtin.com/blockchain/blockchain-healthcare-applications-companies on september 2021.. 
[48] Farmatrust, "Digitising And Innovating The Pharmaceutical And Healthcare Businesses ", Retrieved from URL - https://www.farmatrust.com/ on September 2021.

[49] MTBC, "MTBC Takes Electronic Health Records to the Next Level with Blockchain Technology", Retrieved from URL - https:/www.globenewswire.com/en/newsrelease/2018/06/27/1530349/16541/en/MTBC-Takes-Electronic-Health-Records-to-the-NextLevel-with-Blockchain-Technology.html on september 2021.

[50] Hashed Health, "A Platform for Blockchain Innovation in Healthcare", Retrieved from URL https://hashedhealth.com/ on September 2021.

[51] Sam Daley, "15 Examples of How Blockchain is Reviving Healthcare", Retrieved from URL https://builtin.com/blockchain/blockchain-healthcare-applications-companies on september 2021.

[52] Y. Zhang and J. Wen, "An iot electric business model based on the protocol of bitcoin", Retrieved on July 23, 2019, 18th International Conference on Intelligence in Next Generation Networks (ICIN), 2015, pp 184-191.

[53] Milan Sallaba, Dirk Siegel and Sebastian Becker,"IoT powered by Blockchain", Retrieved from URL - $\quad$ https://www2.deloitte.com/de/de/pages/innovation/contents/iot-powered-byblockchain.html on september 2021.

[54] Sam Daley, "Blockchain and IoT: Examples Making our Future Smarter", Retrieved from https://builtin.com/blockchain/blockchain-iot-examples on september 2021.

[55] Wayra Telefonica Innovation, "Bringing the Supply Chain to the Shopper", Retrieved from URL https://www.wayra.uk/press-releases/provenance-has-been-awarded-the-eus-horizon-2020-1 meufor-blockchains-for-social-good - on september 2021

[56] Sam Mire, BlockchainIn Cybersecurity: 10 Possible Use Cases", Retrieved from https://www.disruptordaily.com/blockchain-use-cases-cyber-security/ on September 2021.

[57] Eric Piscini, David Dalton, and Lory Kehoe, "Blockchain and Cyber Security", Retrieved from URL - https://www2.deloitte.com/content/dam/Deloitte/tr/Documents/technology-mediatelecommunications/Blockchain-and-Cyber.pdf. on September 2021.

[58] Guardtime, "Guardtime Cyber Helping to Achieve Cyber Resilience", Retrieved from URL https://cyber.guardtime.com/ on september 2021.

[59] Remme, "Hot Tech Innovators: Blockchain Based Cybersecurity", Retrieved from URL https://remme.io/ on September 2021.

[60] Blockverify, "Blockchain Based Anti-Counterfeit Solution - Introducing transparency to supply chains", Retrieved from URL - https://pixelplex.io/work/blockchain-supply-chain-and-anticounterfeit-solution/ on September 2021.

[61] Block123, "Building Real-time Frictionless Financial Markets through Trust and Transparency", Retrieved from URL -https://www.block123.com/en/nav/183636233627.html on september 2021.

[62] DavideCarboni. "Feedback based Reputation on top of the Bitcoin Blockchain". In: arXiv preprint arXiv:1502.01504 (2015).

[63] Zibin Zheng 2018, ShaoanXie, Hong-Ning Dai, Xiangping Chen, Huaimin Wang, "Blockchain challenges and opportunities: a survey", International Journal of Web and Grid Services, volume 14, 2018, pp 352-375.

[64] Namecoin, "Namecoin", Retrieved from URL - https://www.namecoin.org/ on September 2021.

[65] Binance Academy, "Blockchain Use Cases: Charity", retrieved from URL https://www.binance.vision/blockchain/blockchain-use-cases-supply-chain, on September 2021.

[66] Theo Goodman, "Using blockchain tokens for royalty payments", retrieved from URL https://medium.com/@theog_/using-blockchain-tokens-for-royalty-payments-964c3037fc54 on september 2021.

[67] Microsoft, "Microsoft launches ambitious blockchain project to help creators get paid", retrieved from URL - https://thenextweb.com/hardfork/2018/06/21/microsoft-and-ey-launch-blockchain-forcopyrights-and-royalties/, on September 2021.

[68] Le Lab, "Solshare, the Startup for Sharing Solar Energy in Bangladesh", retrieved from URL https://medium.com/le-lab/solshare-the-startup-for-sharing-solar-energy-in-bangladesh-en377002b21716, on September 2021. 
[69] GautamDhameja, "UN World Food Programme uses Parity Ethereum to aid 100,000 refugees", retrieved from URL - https://www.parity.io/un-world-food-programme-uses-parity-ethereum-toaid-100-000-refugees/, on September 2021.

[70] Fidelity Charitable, "Fidelity Charitable donors drive a record-breaking year for charitable giving, $\$ 4.5$ billion granted to charity through more than 1 million grants in 2017", retrieved from URL https://www.fidelitycharitable.org/about-us/news/donors-drive-record-breaking-year-forcharitable-giving.html, on September 2021.

[71] C. Amuthadevi, D. S. Vijayan, Varatharajan Ramachandran, "Development of air quality monitoring (AQM) models using different machine learning approaches", Journal of Ambient Intelligence and Humanized Computing, https://doi.org/10.1007/s12652-020-02724-2

[72] LO3Energy, "Reshaping the Energy Future", retrieved from URL - https://lo3energy.com/ on september 2021. 